and endoscopic investigations performed up to 5 years previously were reviewed. Episodes of negative investigations were considered 'missed' opportunities for diagnosis. Clinical outcomes were compared using chi-squared test and Kaplan-Meier survival curves.

Results 396 colorectal cancers were identified with 214 (54\%) males and median age 72 . Of these, 29 (7\%) patients had undergone negative investigations including colonoscopy $(n=8)$, flexible sigmoidoscopy $(n=7)$, barium enema $(n=7)$ and CT for abdominal symptoms $(\mathrm{n}=20)$ ('missed' group) within the previous 5 years, median 817 days prior to diagnosis. Age, mode of presentation, tumour site, $\mathrm{pT}$ and $\mathrm{pN}$ stage were comparable between groups. Metastases at presentation were more common in the 'missed' group ( $28 \%$ vs. $14 \%, p=0.046)$ and survival at median follow-up of 416 days was significantly reduced $(66 \%$ vs. $88 \%, p=0.0004)$.

Conclusion A small proportion (7\%) of patients with colorectal cancer has undergone previous negative abdominal or colonic investigation. Such episodes may represent missed opportunities for diagnosis and survival is significantly reduced in such patients. The recognition that endoscopic and radiological investigations may miss lesions should encourage repeat or alternative interval investigations where concerning symptoms exist.

Disclosure of Interest None Declared.

\section{PTH-014 TWO WEEK WAIT SYMPTOMS ARE PREVALENT IN BOWEL CANCER SCREENING PATIENTS WITH A POSITIVE FAECAL OCCULT BLOOD TEST BUT DO NOT PREDICT CANCER}

doi:10.1136/gutjnl-2013-304907.502

1." P G Vaughan-Shaw, '1 Cutting, 'N R Borley, 'J M Wheeler. 'Department of Colorectal Surgey, Cheltenham General Hospital, Cheltenham, UK

Introduction Studies have reported a high prevalence of lower gastrointestinal (LGI) symptoms in bowel cancer screening (BCSP) patients. However, symptoms are often vague and without characterisation their significance is unclear. This study investigates the prevalence and characteristics of lower gastrointestinal symptoms in screening patients and aims to determine the relevance of two week wait (2ww) symptoms in this cohort.

Methods A prospective cohort study was performed. BCSP patients presenting for colonoscopy over a 7-month period were included. Data on symptom prevalence, frequency and duration was collected and assessed against 2 -week wait criteria. Associations between symptom prevalence and outcome were investigated using the two-tailed $\chi^{2}$ test.

Results Symptom and outcome data was collected in 397 patients. LGI symptoms were reported by 282 (71\%) patients and 37 patients $(9 \%)$ were found to have colorectal cancer (CRC). Symptom prevalence was comparable between those with or without CRC $(65 \%$ vs. $72 \%, \mathrm{p}=0.473)$. Meanwhile, $2 \mathrm{ww}$ symptoms were reported in $148(37 \%)$ of all patients. $2 \mathrm{ww}$ symptom prevalence was comparable in those with and without cancer (38\% vs. 39\%, p = 0.915).

Conclusion This study demonstrates that while $2 \mathrm{ww}$ symptoms are highly prevalent in a FOB positive cohort, they do not predict a finding of colorectal cancer. These findings suggest that $2 \mathrm{ww}$ symptoms could not be used to prioritise investigation in this cohort while in those patients referred with $2 \mathrm{ww}$ symptoms, additional FOB testing would offer little predictive utility. Further efforts to increase public awareness of cancer symptoms are required, whilst false reassurance from a negative result should be discouraged.

Disclosure of Interest None Declared.

\section{PTH-015 PROSPECTIVE COMPARISON OF FICE AND I-SCAN FOR IN- VIVO CHARACTERISATION OF SMALL COLONIC POLYPS}

doi:10.1136/gutjnl-2013-304907.503

1."P J Basford, 'G R Longcroft-Wheaton, 'P Bhandari. 'Gastroenterology, Portsmouth hospitals NHS trust, Portsmouth, UK
Introduction In-vivo characterisation of small colonic polyps has been reported using several technologies but with few prospective comparisons between them. We aimed to compare the accuracy of Flexible Spectral Imaging Colour Enhancement (FICE) and i-Scan in the assessment of polyps $<10 \mathrm{~mm}$. In addition the relationship between accuracy of white light assessment (WL) and resolution of endoscope was assessed.

Methods Patients undergoing screening colonoscopy through the UK BCSP were prospectively recruited. All procedures were performed by a single endoscopist with extensive experience in invivo diagnosis. For the FICE group Fujinon 410,000 pixel CCD and 650,000 pixel CCD colonoscopes were used with an EPX 4400 processor. For the i-Scan group Pentax 1.2 Megapixel colonoscopes were used with an EPKi processor. All polyps $<10 \mathrm{~mm}$ were assessed sequentially using white light endoscopy (WL) and either FICE or i-Scan before resection. Predicted histology was recorded with both modalities and compared to the final histopathological diagnosis. In-vivo characterisation accuracy was analysed based on the resolution of the endoscopes used; standard definition - SD (410K pixel), high definition - HD (650K pixel) and $\mathrm{HD}+$ (1.2M pixel).

Results In the FICE group 293 polyps of mean size $4.7 \mathrm{~mm}$ were assessed in 170 patients. In the i-Scan group 209 polyps of mean size $4.3 \mathrm{~mm}$ were assessed in 84 patients. There was no significant difference in WL accuracy between SD and HD endoscopes (70\% vs $72.7 \%, p=0.606$ ), however accuracy was significantly higher with the HD+1.2megapixel CCD endoscopes (93.3\%) compared to both the SD $(70.0 \%, p=0.0001)$ and $\mathrm{HD}(72.7 \%, p=0.0001)$ endoscopes. Sensitivity was significantly greater with FICE using an HD endoscope compared to an SD endoscope (92.6\% vs $83.3 \%$, $p=0.048)$. Overall accuracy was significantly greater with $\mathrm{HD}+\mathrm{i}-\mathrm{Scan}(94.7 \%)$ than SD FICE $(82.7 \%, p=0.0003)$ and HD FICE $(88.8 \%, p=0.0439)$. The use of FICE improved accuracy from $70.0 \%$ with WL to $82.7 \%$ $(p=0.014)$ and from $72.7 \%$ with WL to $88.8 \%(p<0.001)$ for SD and $\mathrm{HD}$ endoscopes respectively. Only a minor gain over WL was seen with addition of iScan $(93.3 \%$ to $94.7 \%, p=0.68)$.

\section{Abstract PTH-015 Table 1}

\begin{tabular}{lccc}
\hline & Sensitivity \% & Specificity $\%$ & Accuracy \% \\
\hline SD WL & 76.0 & 59.3 & 70.0 \\
HD WL & 75.8 & 66.7 & 72.7 \\
HD+ WL & 95.5 & 89.3 & 93.3 \\
SD FICE & 83.3 & 81.5 & 82.7 \\
HD FICE & 92.6 & 81.3 & 88.8 \\
HD+ i-Scan & 97.0 & 90.7 & 94.7 \\
\hline
\end{tabular}

\section{Conclusion}

1. Only a small, non-significant gain in WL accuracy is seen between a $410 \mathrm{~K}$ pixel SD endoscope and a $650 \mathrm{~K}$ pixel HD endoscope. However diagnostic accuracy with WL improves significantly with a 1.2 megapixel endoscope.

2. FICE significantly improves accuracy when used with an $\mathrm{SD}$ or HD endoscope but the very high WL accuracy of a 1.2 megapixel endoscope allows no significant additional improvement with i-Scan.

Disclosure of Interest None Declared.

\section{PTH-016 DEFECATING SCINTIGRAPHIC PHOTOGRAPHY AT ST GEORGE'S HOSPITAL: REVIEW OF FINDINGS AND CORRELATION WITH PATIENT SYMPTOMS}

doi:10.1136/gutjnl-2013-304907.504

1.*R I Rusu, ${ }^{2} \mathrm{~N}$ Beharry, ${ }^{3} \mathrm{~A}$ Irwin, ${ }^{4} \mathrm{~S}$ Heenan, ${ }^{1} \mathrm{~A}$ Poullis. ${ }^{1}$ Gastroenterology Department, St George's Hospital; ${ }^{2}$ Radiology, St. George's Hospital; ${ }^{3}$ Medical Physics; ${ }^{4}$ Radiology, St George's Hospital, London, UK 
Introduction Defecating scintigraphic proctograms (DSPs) have an established position in the investigation of evacuatory problems and ano-rectal symptoms. Functional radionuclide imaging allows for both the assessment of rectoceles and correlation with evacuatory function.

Methods 151 consecutive DSPs carried out over a 3-year period were reviewed. Clinical details of presenting symptoms were reviewed with the study results. All studies were reviewed for evidence of anatomical abnormalities and function via $\%$ excretion. The $\%$ excretion was analysed in those with and without a rectocele and compared with symptoms (constipation, incontinence, rectal pain and evacuatory difficulty). The mean $\%$ excretion for each symptom (present/absent) was calculated and compared with a t test.

Results Of the 151 patients, 23 were unable to defecate and no results were available. There were 8 males, 143 females. Mean age range $55+/-14.76 \%$ had a rectocele demonstrated. In all patients $\%$ excretion was significantly different in those with symptomatic evacuatory difficulty (\% excretion: Constipation present/absent $63 \% \mathrm{v}$ $62.6 \% p=0.9$, Incontinence present/absent $60.9 \%$ v $63.4 \% p=0.46$, Pain present/absent $67.2 \%$ v $62.1 \%, p=0.24$, Evacuatory difficulty present $/ a b s e n t 60.2 \% \vee 66.3 \% \mathrm{p}=0.03$ ). Of those with a rectocele there was a non-significant trend to abnormality on excretory function (\% excretion: Constipation present/absent $62.8 \%$ v $61.8 \%$ $p=0.8$, Incontinence present $/$ absent $60.9 \%$ v $62.6 \% p=0.6$, Pain present/absent $66.6 \%$ v $61.5 \%, p=0.33$, Evacuatory difficulty present/ absent $59.7 \%$ v $65.9 \% \mathrm{p}=0.06$ ). On those without a rectocele, there was no difference in excretory function in any symptom groups.

Conclusion In this large series DSPs identified rectoceles in $76 \%$ of studies. In those with a rectocele functional impairment was often present, with a trend to reduced \% excretion seen. In those without a rectocele \% excretion on DSPs did not differ in any symptom group. Larger reviews are needed to identify small sub-groups who may benefit from this study. The significant number with abnormalities found on DSPs suggests that this investigation may be underutilised in those with ano-rectal symptoms.

Disclosure of Interest None Declared.

\section{PTH-017 STRATEGY FOR COLORECTAL CANCER (CRC) SCREENING IN INDIVIDUALS WITH SIGNIFICANT CO-MORBID CONDITIONS}

doi:10.1136/gutjnl-2013-304907.505

1."R Ramaraj, ${ }^{2} \mathrm{R}$ Dixon, ${ }^{2 \mathrm{H}}$ Adams, ${ }^{1} \mathrm{~S}$ Dolwani. ${ }^{1}$ Department of Gastroenterology; ${ }^{2} \mathrm{De}-$ partment of Radiology, Llandough hospital, Cardiff and Vale UHB, Cardiff, UK

Introduction With the advent of the Bowel cancer screening programme (BCSP) in the UK participants who have positive FOB tests are generally offered optical colonoscopy $(\mathrm{OC})$ if considered safe, for early detection and prevention of CRC. In our study we undertook Computed Tomographic Colonography (CTC) in our hospital as the investigation of choice in those FOBt positive individuals with an American Society of Anesthesiology (ASA)grade $>=3$ and analysed outcomes.

Methods Cases were prospectively discussed with screening practitioners carrying out assessments prior to $\mathrm{OC}$ and on the basis of hospital records and liaison with primary care physicians were assigned to CTC/OC with majority proceeding to OC as per standard practise. Patient data was accessed from our database in radiology, endoscopy and histology retrospectively and ASA grade assigned on the basis of the above information.44/69 cases that were referred for CTC from the BCSP between Feb 2009 and Nov 2011, were considered to have an ASA > = 3.CTC results were analysed and correlated with endoscopic and histological findings.

Results Out of 44 cases (17 female,27 male, mean age 65.4) with positive FOBt referred for CTC in the BCSP, $50 \%(22)$ of patients had positive findings i.e.18 polyps and 4 suspected CRC.3/44 cases had a normal Flexible Sigmoidoscopy (FS) before CTC. Hence 41 of the above were considered to have had a primary CTC.44\% (18/41) of patients referred for primary CTC had an endoscopy (20\% OC,24\% FS), of which $61 \%$ had a polypectomy. 41 primary CTCs detected 32 polyps in 18 cases and 4 cancers. 18 cases (4 diminutive polyps on CTC not requiring OC/FS) underwent endoscopy as a result of the above (OC 8, FS 10) detecting 36 polyps (35 removed) in 11 patients. $86 \%$ of polyps were detected on the left side and the majority of this (83\%) were histologically confirmed to be adenomas. In addition to this 2 left sided cancers was confirmed endoscopically.

Conclusion In this small cohort CTC seems comparable to colonoscopy for detection of polyps and cancers ${ }^{1}$. In the patient group selected almost $44 \%$ of cases thought to have significant comorbidity who had primary CTC ended up having a lower GI endoscopy along with a need for therapy. It is also noted that the majority of significant polyps and all cancers were located in the left colon. We suggest that in this group a larger study evaluating a combination of CTC with FS (with no or minimal sedation) would be most appropriate in the context of the BCSP. It may also be useful to have evidence based criteria on fitness for colonoscopy in order to inform individuals and programmes on the appropriateness of screening in the context of comorbidity and the risk to benefit ratio.

Disclosure of Interest None Declared.

\section{REFERENCE}

1. Laghi, A et al. Current status on performance of CTC and clinical indications. EJR. doi:10.1016/j.ejrad.2012.05.026

\section{PTH-018 TOWARDS NOVEL NON INVASIVE METHODS TO DIAGNOSE COLORECTAL CANCER USING AN ELECTRONIC NOSES (E-NOSE) AND FIELD ASYMMETRIC ION MOBILITY SPECTROMETRY (FAIMS)}

doi:10.1136/gutjnl-2013-304907.506

${ }^{1} \mathrm{~J}$ Covington, ${ }^{2} \mathrm{E}$ Westinbrink, ${ }^{3} \mathrm{~N}$ O'Connell, ${ }^{3} \mathrm{C}$ Bailey, ${ }^{4} \mathrm{~S}$ Smith, ${ }^{3} \mathrm{C}$ Nwokolo, ${ }^{5} \mathrm{C}$ Harmston, ${ }^{6} \mathrm{~K}$ Bardhan, ${ }^{7, *} \mathrm{R}$ Arasaradnam. ' ${ }^{1} \mathrm{School}$ of Engineering; ' 2 niversity of Warwick; ${ }^{3}$ University Hospital Coventry \& Warwickshire, Coventry, UK; ${ }^{4}$ Biochemistry: ${ }^{5}$ Colorectal Surgery, University Hospital Coventry \& Warwickshire, Coventry; ${ }^{6}$ Rotherham NHS Foundation Trust, Rotherham; ' Clinical Sciences Research Institute, University of Warwick, Coventry, UK

Introduction Using an electronic nose (E-nose) we have previously demonstrated its ability to detect inflammatory bowel disease (IBD) by shifts in the patterns of volatile organic compounds (VOCs) in the gases and vapours that emanate from urine samples. A similar distinction could be made using FAIMS, which involves a different principle, but still with gas phase samples. Here, we have extended our work to detect colon cancer from odours from urine alone.

Methods Technology Principles: The E-nose uses an array of gas phase chemical sensors which are broadly tuned to different chemical groups (e.g. alcohols, gases). When a sample is presented to the sensor array, as each sensor is different, it will produce a unique response to that sample. By taking all of the sensor responses together, we can create a 'bio-odorant fingerprint' of that sample; thus mimicking the human olfactory system. FAIMS operates on similar principles, but produces its fingerprint by measuring the differences in mobility of ionised chemicals in high electric fields. 47 subjects were recruited; 20 with colonic adenocarcinoma (CRC) and 27 controls. The latter comprised 20 with ulcerative colitis (UC) in remission (defined as SCAI score $<4$ ) and 7 healthy subjects. $10 \mathrm{ml}$ urine aliquots were collected and stored frozen. For assay, the containers were first heated to $60 \pm 0.1 \mathrm{oC}$. The headspace (the air above the sample) was analysed by an AlphaMOS FOX 4000 E-nose and by an Owlstone Lonestar FAIMS instrument. Discriminant Function Analysis and Fisher Discriminant Analysis were used for statistical evaluation, respectively.

Results The E-nose (Figure 1) and FAIMS plots (not shown) shows those with CRC are tightly grouped and distinct from healthy controls and those with UC $(p<0.001)$. 